\title{
Molecular Docking and Molecular Dynamics Studies of Role of Anti-Allergic Medicines as Inhibitors Against Covid-19
}

Mrinal Kanti Si ( $\sim$ mrinalchem@gmail.com )

Sardar Patel University

\section{Research Article}

Keywords: Molecular docking, Anti-allergic medicines, Inhibitor, non-covalent interaction, Covid-19, vaccines, Ebola

Posted Date: January 7th, 2022

DOI: https://doi.org/10.21203/rs.3.rs-1192723/v1

License: (c) (1) This work is licensed under a Creative Commons Attribution 4.0 International License.

Read Full License 


\section{Abstract}

The 2019-nCoV virus is a human-infectious coronavirus (CoV). Very few treatment options are available to healthcare professionals who are fighting this outbreak at the front. The main warning symptoms of COVID-19, the disease caused by the new coronavirus, are fever, fatigue, and a dry cough, sometimes it also causes cold-like symptoms like a runny nose which are sometimes similar to symptoms of allergies and sometimes difficult to differentiate between COVID-19 and allergies. The anti-allergic drug molecules can behave as good inhibitor against COVID-19. Molecular docking studies have been performed to examine the inhibitor properties of anti-allergic molecules against Covid-19. The searching of better inhibitors have been examined interns of various non-covalent interactions like hydrogen bond, halogen bond, vander waal's interactions, alkyl-паnd $\pi-\pi$ interactions between small molecules (Anti-allergic medicines) with main protease of Covid-19 using molecular docking and Molecular Dynamics simulation which reveals that astemizole is best inhibitor among ten Anti-allergic drug molecules.

\section{Introduction:}

The 2019-nCoV (2019 novel coronavirus) is one of newly emerged coronavirus which originated in a Wuhan sea food market of china and has quickly spread in the rest of world. ${ }^{1}$ The 2019 -nCoV virus is a human-infectious coronavirus (CoV) [1, 2]. Very few treatment options are available to healthcare professionals who are fighting this outbreak at the front. The possible identification treatment become easy after determination of genome sequence (GenBank ID: MN908947.3) [2]. At this moment, researchers have involved to develop the vaccines, small molecules, and biological therapeutics to target specifically 2019-nCoV virus which is essential to benefit patients in the current outbreak. However, the sequences of 2019-nCoV (2019-novel Corona Virus) matches 82\% with respiratory syndrome-related coronavirus (SARS-CoV, GenBank ID: NC_004718.3). Therefore, the knowledge of medicinal chemistry studies on SARS-CoV and the Middle East Respiratory Syndrome (MERS-CoV) may help us to find the specific medicine for treatment 2019-nCoV [3]. The spike proteins of CoV binds a host cell-surface receptor for entry and for 2019-nCoV, the receptor is angiotensin-converting enzyme 2 (ACE2) [4]. The positive genomic RNA of virus attaches directly to the host ribosome inside the host cell for the translation of two large conterminal poly proteins through the process proteolysis into components for packaging new virions [5]. The proteolysis process generates the two proteases which are coronavirus main proteinase (3CLpro) and the papain-like protease (PLpro) [6]. CoV encodes another proteins replicase that is an RNA-dependent RNA polymerase (RdRp) which can help to replicate the RNA genome [7]. Researcher can targets these four proteins like spike, RdRp, 3CLpro, and PLpro for treatment of for 2019-nCoV [8].

The computer-aided drug design techniques have been efficiently used to quickly identify promising drug repurposing candidates. That technique becomes easy after resolving the detailed 3D-structures of key virous proteins. Recently, the crystal structure of 2019-nCoV protease in complex with a covalentlybonded inhibitor, N3 (PDB: 6LU7) have been reported which will give advantage to design the proper 
inhibitor i.e. small molecule for 2019-nCoV $[9,10]$. There are few reports on the study of inhibitors of 2019-nCoV.

There are some reports on the several promising known drugs stand out as potential inhibitors of 2019$\mathrm{nCoV}$ protease. The ritonavir and remdesivir showed efficacy at cellular level [11-14]. However, remdesivir has been previously used to treat Ebola virus. The studies of Oseltamivir (prodrug of oseltamivir carboxylate), drug have also been performed against SARS-CoV-2 which is commonly used to treat influenza viruse [15]. Arbidol (with an antiviral effect of $100 \%$ after 14 days of treatment) and lopinavir/ritonavir (with an antiviral effect of $55.9 \%$ after 14 days of treatment) also can be used significantly against anti-SARS-CoV-2 effect [16]. Significant anti-SARS-CoV-2 effect has been shown by Darunavir and hydroxychloroquine [17]. The anti-SARS-CoV-2 effects have examined using favipiravir (prodrug of favipiravir-4-ribofuranosyl-5'-monophosphate and favipiravir-4-ribofuranosyl-5'-triphosphate, $\mathrm{EC} 50=61.88 \mu \mathrm{M}, \mathrm{CC} 50>400 \mu \mathrm{M}, \mathrm{SI}>6.46$ ), [18] ribavirin (prodrug of ribavirin 5'-monophosphate, $\mathrm{EC50}=$ $109.50 \mu \mathrm{M}, \mathrm{CC} 50>400 \mu \mathrm{M}, \mathrm{SI}>3.65)[19,20]$ nitazoxanide (prodrug of tizoxanide, EC50 $=2.12 \mu \mathrm{M}, \mathrm{CC} 50$ $>35.53 \mu \mathrm{M}, \mathrm{SI}>16.76)[21,22]$ and chloroquine $(E C 50=1.13 \mu \mathrm{M}, \mathrm{CC} 50>100 \mu \mathrm{M}, \mathrm{SI}>88.50[12,23]$. The computational studies have been also examined to observe anti-SARS-CoV-2 effects with saquinavir, remdesivir,dolutegravir, and bictegravir [24].

There are some similarity symptoms of Covid-19 and allergy. The main warning symptoms of COVID-19, the disease caused by the new coronavirus, are fever, fatigue, and a dry cough, sometimes, it also causes cold-like symptoms like a runny nose. Therefore, it may be sometimes difficult to differentiate between COVID-19 and allergies in allergy season. Severe allergies can make you can feel tightness in your chest and shortness of breath, especially if you have asthma, too which are also serious symptoms of COVID19 [25].

In this article, we have searched ten Anti-allergic molecules (Astemizole, Clemastine, Cyeproheptadine, Fluorometholone, Flucasoni, lodoxiamide, Loratadine, Nedocromil, Olopatadine, and Triprolidine) for the study of interactions with main protease of Covid-19. The interactions studies and interaction energies between Anti-allergic molecules and main protease of Covid-19 have been performed using molecular docking which have been collected from the previously reported crystal structure of inhibiter-protein complex (PDB: 6LU7). The Molecular Dynamics Simulation studies also been performed using Gromacs which result also corroborates with docking studies.

\section{Computational Methods:}

The docking study has been performed using AutoDock Vina as docking software. 10 Anti-allergic medicines (ligands) have been searched to examine inhibitor properties against Covid-19 [26]. The ligand molecules and the crystal structure for SARS-CoV-2 was taken from Protein Data Bank (PDB: 6LU7) and missing residues of SARS-CoV2 were added with Swiss Model [27-29]. Before docking study we have added the hydrogen in ligand molecules and main protease of Covid-19. The anti-allergic molecules were docked with the active of main protease of Covid-19 where the active site has been considered from the 
previously reported crystal structure of complex of inhibitor-main protease of Covid-19 (PDB: 6LU7) using Pyrex virtual screening tool using AutoDock Vina $[30,31]$.The results of screening were analyzed with Discovery Studio visualization software [32].

\section{Molecular docking and ranking:}

AutoDock Vina (Trott \& Olson 2010) is an open-source program, which was used for molecular docking of ligands. Initially, AutoDockTools has been used for all inhibitors and energy minimized proteins (with or without binding site water residues) for the conversion into PDBQT files. GridBox was generated for each protein-inhibitor complexes and configuration files were created for each docking run. The exhaustiveness has been set to 10 for all run. The Lamarckian genetic algorithm has been used as a search engine. AutoDock 4.0 force field has been used [33].

\section{Simulation:}

For molecular dynamics (MD) simulations, we have taken the docking structure of Asetemizole with main protease of Covid-19 and crystal structure of Covid-19 during 25 ns time scale. GROMACS 4.6.3 has been used for molecular dynamics simulations using with AMBER 03 force field [34, 35]. For MD simulation, the Aseemizole-Covid-19 complex and Covid-19 protein were soaked in cubic box filled with TIP4P water molecules at a distance of $10 \AA$ away from the box wall. Then the energy minimization step has been performed energy minimization to remove steric clashes between solvent molecules which involved 2000 000 steps during the time period of 1000 ps. Position restrained dynamics at a constant volume and temperature (NVT) was carried out for 200 ps where the temperature was raised to $300 \mathrm{~K}$ with a coupling time of $0.1 \mathrm{ps}$. And then NPT has been performed at $300 \mathrm{~K}$ for $200 \mathrm{ps}$ using a Berendsen barostat and a V-rescale thermostat coupling with a coupling time of 0.1 ps $[34,36,37]$. For monitoring pressure we have employed the Berendsen coupling method.

\section{Results And Discussion:}

In this article, we have selected the ten anti allergetic drug molecules (Astemizole, Clemastine, Cyeproheptadine, Fluorometholone, Flucasoni, lodoxiamide, Loratadine, Nedocromil, Olopatadine, and Triprolidine) to examine the activity for the treatment of Covid-19 (Figure 1).

The several anti allergetic drug molecules are docked in the active site of COVID-19 main protease (PDB: 6LU7). Initially, we have removed the inhibitor from the active site of crystal structure (PDB: 6LU7). The similar interactions are observed for the docked inhibitor on the active site of COVID-19 main protease and crystal structure with crystal structure (Figure 2). Further, we have docked the ten anti allergetic drug molecules with the active site of COVID-19 main protease.

The binding energies calculated in docking study are given in Table 1. The interaction energies in the active site of inhibitor (N3-4) is $7.9 \mathrm{kcal} / \mathrm{mol}$. Further, we have calculated interaction energies of other anti-allergic molecules with the active site of COVID-19 main protease using Docking Study. 
Table 1

Table for interaction energies between inhibitor and Covid-19 protein calculated in Docking Study.

\begin{tabular}{llllll} 
S. No. & Drug Molecules & Energy $(\mathrm{kcal} / \mathrm{mol})$. & S. No. & Drug Molecules & Energy \\
\hline 1 & N3-4 & -7.9 & 7 & lodoxiamide & -6.8 \\
\hline 2 & Astemizole & -8.1 & 8 & loratadine & -7.3 \\
\hline 3 & Clemastine & -6.6 & 9 & Nedocromil & -7.4 \\
\hline 4 & Cyproheptadine & -6.6 & 10 & Olopatadine & -7.4 \\
\hline 5 & Fluorometholone & -7.1 & 11 & Triprolidine & -7.4 \\
\hline 6 & Fluticasoni & -7.2 & & & \\
\hline
\end{tabular}

Inhibitor N3 make similar interaction with docking structure. Crystal structure analysis shows that inhibitor N3 make the interaction with various active residues like MET-165, GLU-166, GLY-143, GLN-189, THR-190, ALA-191, PRO-168, HIS-172, PHE-140, LIU-167, HIS-41, MET-49. Further, we have again docked same inhibitor in the active site of COVID-19 main protease. Docking structure analysis reveals that inhibitor interacts with the nearly similar active site like crystal structure (MET-165, GLU-166, GLY-143, GLN-189, THR-190, ALA-191, HIS-163, SER-144, CYS-145) (Figure 2).

Further, we have docked other ten anti-allergic drug molecules (Astemizole, Clemastine, Cyeproheptadine, Fluorometholone, Flucasoni, lodoxiamide, Loratadine, Nedocromil, Olopatadine, and Triprolidine) with the active site of main protease of Covid-19.

Interaction energies analysis from docking study reveals that Astemezole interacts strongly even greater than inhibitor N3 where the interaction energy is $8.1 \mathrm{Kcal} / \mathrm{mol}$ (Table 1 \& Figure 3 ). The Astemezole interacts with the residues of THR-26, HIS-41, PHE-140, LEU-141, ASN-142, GLY-143, CIS-145 and MET-165 of Covid-19 main Protease (Figure 3). Numbers of interactions present in astemezole-protein complex are less than that of inhibitor N3. However, $\pi-\pi$ stacking interactions and halogen bonding can play the important role for stronger interaction compare to inhibitor N3. anti-allergic clamastine interacts with active site of protein weakly among the above molecules (Figure 1 \& Figure 4, Table 1). The analysis shows that the numbers of interaction present in the complex of clamastine and main protease of Covid19 are less. Clamastine interacts with active residues of ASN-142, LEU-27, CYS-145 and MET-165 of main protease of Covid-19 (Figure 4).

Crypoheptadiene interacts with active sites of main protease of Covid-19 is similar to that of Clemastine (interaction energy is $6.6 \mathrm{kcal} / \mathrm{mol}$ ). Similar type of interactions is present in both of clemastine and crypoheptadine molecule (Alkyl- $\pi, \pi-\pi$ and vanderwaal's interactions) which results corroborates with interactions energies (Table 1, Figure 4 and Figure 5).

lodoxamide 
The interaction energies obtained from docking study reveals that lodoxamide, Fluorometholone, and Fluticasoni are nearly same (lodoxamide $=6.8 \mathrm{Kcal} / \mathrm{mol}$; Fluorometholone $=7.1 \mathrm{Kcal} / \mathrm{mol}$.; Fluticasoni $=$ $7.2 \mathrm{Kcal} / \mathrm{mol}$.) (Table 1). The numbers of interactions as well as type of these interactions present in the complex of Fluorometholone, Fluticasoni and lodoxamide with main protease of Covid-19 are also similar (Figure 6, 7 and 8).

loratadiene, Nedocromil, Olopatadiene and Triprolidine interaction stronger compare to Fluorometholone, Fluticasoni and lodoxamide.

\section{Md Simulation:}

Further, we have performed Molecular Dynamics simulation using best inhibitor Astemizole with main protease of Covid-19 for 25 ns. Total hydrogen bonding, root-mean-square deviation (RMSD), root mean square fluctuation (RMSF) and radius of gyration have been examined during Molecular Dynamics Simulation for $25 \mathrm{~ns}$ for the complex of inhibitor (Astemizole)- Covid-19 protein and crystal structure of Covid-19 protein (Figure 10,11, 12 and 13).

Total hydrogen bonding analysis reveals that inhibitor Astemizole interacts with active site of main protease of Covid-19 during 25 ns which corroborates with docking study (Figure 10). We have extended our study to analyze root-mean-square deviation (RMSD), root mean square fluctuation (RMSF) and radius of gyration to examine the stability of covid-19 protease during interaction with inhibitor Astemizole.

In figure 11, we presented detailed analysis for the RMSD as a function of time of the various part of the Covid-19 protein-Astemizole complex (Figure 11 (a)) and Covid-19 protein (Figure 11(b)). RMSD value of various residues are changed little even in presence of Astemizole which suggest that protein is stable after interaction with inhibitor.

RMSF stands for root mean square fluctuation. This is a numerical measurement similar to RMSD, but instead of indicating positional differences between entire structures over time, RMSF is a calculation of individual residue flexibility, or how much a particular residue moves (fluctuates) during a simulation. RDMF values for Astemizole-protein complex and Covid-19 protein suggests that residues present in Astemizole-protein complex are effected little even interaction with inhibitor Astemizole. Therefore, Protein is stabilized in interaction with inhibitor Astemizole (Figure 12).

Our studies are further extended with calculation of radius of gyration which indicates the compactness of proteins which in turn reflects its stability. It plays a significant role during comparative study as fluctuates the less it is stable at that point of time. We have calculated the Radius of Gyration of Covid-19 protein and Complex of Covid-19 and Astemizole. The results suggests that Protein is stable even interaction with Astemizole (Figure 13). 
We have calculated the electrostatic and van der Waals interaction energies between Astemizole inhibitor and Covid-19 protein to examine the nature of interactions during MD simulation. The interactions studies reveal that Van der Waals interactions play vital role stabilize the Covid-19 protein with Astemizole inhibitor (Figure 14). Figure 14, shows that Astemizole inhibitor stabilize main protease of Covid-19 in between 10 ns to 15 ns.

\section{Conclusion:}

The American College of Allergy, Asthma \& Immunology (ACAAI) is providing direction on the continued use of corticosteroids for patients with allergies and asthma during the COVID-19 pandemic. According to American College of Allergy, Asthma \& Immunology (ACAAI), it is more important use of corticosteroids to keep symptoms well controlled for these patients who are suffering spring allergy season, allergy and asthma. Therefore, anti allergetic medicines may be important for the treatment of Covid-19 patient and these may be better inhibitors for main protease of Covid-19. This study reveals that ten anti allergetic medicines are interacted with the main protease of Covid-19 using computation studies. The searching of better inhibitors have been examined interns of various non-covalent interactions like hydrogen bond, halogen bond, vander waal's interactions, alkyl- $\pi$ and $\pi-\pi$ interactions between small molecules (antiallergic medicines) with main protease of Covid-19. Our studies reveal that Astemizole interacts strongly among these ten molecules and even better than the inhibiters which crystal structure with main protease of Covid-19 is reported in the literature [9]. Other molecules like Nedocromil, Olopatadine and Triprolidine are also better inhibitor against main protease of Covid-19. Clemastine and Cyproheptadine interact weaker among ten anti-allergic medicines. Therefore, our studies help us to choice as well as design of anti-allergic medicines properly for the treatment Covid-19.

\section{Declarations}

\section{Funding:}

There is no funding for this publication.

\section{Conflict of interest:}

The author declares that there is no conflict of interest.

\section{Availability of data and material:}

There is no data and material available for this publication.

\section{Code availability:}

There is no code available for this publication.

\section{Authors' contributions:}


Author has designed, performed and written the manuscript

\section{Acknowledgement:}

MKS thanks to Sardar Patel University, Balaghat, M.P, and India for giving opportunity for research. I thank to Dr. Kalyanashis Jana CSIR-CSMCRI for his help.

\section{References}

[1] Zhou P, Yang XL, Wang XG, Hu B, Zhang L, Zhang W, Si HR, Zhu Y, Li B, Huang CL, Chen HD, Chen JY, Luo, Guo H, Jiang RD, Liu MQ, Chen Y, Shen XR, Wang X, Zheng XS, Zhao K, Chen QJ, Deng F, Liu LL, Yan B, Zhan FX, Wang YY, Xiao G. \& Shi ZA (2020). pneumonia outbreak associated with a new coronavirus of probable bat origin. Nature 579: 270-273.

[2] Morse JS, Lalonde TS, Xu \& Ray, Liu W (2020) Treatment Options for Severe Acute Respiratory Infections Caused by 2019-nCoV. ChemBioChem. 21:730 - 738

[3] Li F, Li W, Farzan M, Harrison SC (2005) Structure of SARS coronavirus spike receptor-binding domain complexed with receptor. Science. 309:1864-1868.

[4] Dong N, Yang X, Ye L, Chen K, Chan E, Yang M, Chen S (2020) Genomic and protein structure modelling analysis depicts the origin and infectivity of 2019-nCoV, a new coronavirus which caused a pneumonia outbreak in Wuhan, China, bioRxiv, https://www.biorxiv.org/content/10.1101/2020.01.20.913368v2 (accessed Jan. 25, 2020).

[5] Baranov P, Henderson VCM, Anderson CB, Gesteland RF, Atkins JF, Howard MT (2005) Programmed ribosomal frameshifting in decoding the SARS-CoV genome. Virology. 332:498-510.

[6] Ziebuhr J, Snijder EJ, Gorbalenya AE, (2000) Virus-encoded proteinases and proteolytic processing in the Nidovirales. J. Gen. Virol. 81:853 -879.

[7] Xu X, Liu Y, Weiss S, Arnold E, Sarafianos SG, Ding J (2003) Molecular model of SARS coronavirus polymerase: implications for biochemical functions and drug design, Nucleic Acids Res. 31:7117-7130.

[8] Li CC, Wang XJ, Wang HCR (2019) Repurposing host-based therapeutics to control coronavirus and influenza virus. Drug Discovery Today 24:726 -736.

[9] Liu X, Zhang B, Jin Z, Yang H, Rao Z The crystal structure of COVID-19 main protease in complex with an inhibitor N3, [10.2210/pdb6LU7/pdb]

[10] Al-Osai AM, Al-WazzM J ah. (2017) The history and epidemiology of Middle East respiratory syndrome corona virus. Multidiscip Respir Med. 12:20. (doi: 10.1186/s40248-017-0101-8) 
[11] Prajapat M, Sarma P, Shekhar N, Avti P, Sinha S, Kaur H, Kumar S, Bhattacharyya A, Kumar H, Bansal S, Medhi B (2020) Drug targets for corona virus: A systematic review, Indian Journal of Pharmacology. 52:56-65.

[12] Gralinski L, Menachery V (2020) Return of the Coronavirus: 2019-nCoV. Viruses. 24:135.

[13] Roosa S, Tikkanen MPH, Res M, Eric C, Schneider M.D.(2021) Social Spending to Improve Population Health - Does the United States Spend as Wisely as Other Countries? The New England journal of medicine. 382:885-887.

[14] Liang C, Tian L, Liu Y, Hui N, Qiao G, Li H (2020) A promising antiviral candidate drug for the COVID19 pandemic: A mini-review of remdesivir. European journal of medicinal chemistry. 201:112527.

[15] Lu H (2020) Drug treatment options for the 2019-new coronavirus (2019-nCoV). Bioscience trends. 14:69-71.

[16] Zhu Z, Lu Z, Xu T, Chen C, Yang G, Zha T, et al. (2020) Arbidol monotherapy is superior to lopinavir/ritonavir in treating COVID-19. The Journal of infection. 81:e21-e3.

[17] Spezzani V, Piunno A, Iselin H (2020) Benign COVID-19 in an immunocompromised cancer patientthe case of a married couple. Swiss medical weekly. 150:w20246.

[18] Furuta Y, Takahashi K, Shiraki K, Sakamoto K, Smee D, Barnard et al. (2009)T-705 (favipiravir) and related compounds: Novel broad-spectrum inhibitors of RNA viral infections. Antiviral research, 82:95102.

[19] Ferron F, Subissi L, Silveira De Morais AT, Le, NTT,. Sevajol M, Gluais L, et al.(2018) Structural and molecular basis of mismatch correction and ribavirin excision from coronavirus RNA. Proceedings of the National Academy of Sciences. 115:E162-e71.

[20] Krajczyk A, Kulinska K, Kulinski T, Hurst BL, Day CW, Smee DF et al.(2014) Antivirally active ribavirin analogues-4,5-disubstituted 1,2,3-triazole nucleosides: biological evaluation against certain respiratory viruses and computational modelling. Antivir Chem Chemother. 23:161-71.

[21] Tilmanis DC, van Baalen, Oh DY, Rossignol JF, Hurt AC, (2017) The susceptibility of circulating human influenza viruses to tizoxanide, the active metabolite of nitazoxanide. Antiviral Res.147:142-148.

[22] Pepperrell T, Pilkington V, Owen A, Wang J, Hill AM, (2020). Review of safety and minimum pricing of nitazoxanide for potential treatment of COVID-19. Journal of virus eradication.6:52-60.

[23] Fantini J, Di Scala C, Chahinian H, Yahi N. (2020). Structural and molecular modelling studies reveal a new mechanism of action of chloroquine and hydroxychloroquine against SARS-CoV-2 infection. Int $\mathrm{J}$ Antimicrob Agents. 55:105960. 
[24] Jr. Hall DC, Ji HF (2020). A search for medications to treat COVID-19 via in silico molecular docking models of the SARS-CoV-2 spike glycoprotein and 3CL protease. Travel Med Infect Dis.35:101646.

[25] Allergy Symptoms vs. Coronavirus (COVID-19), WebMD, [https://www.webmd.com/lung/

covid-allergies]

[26] Shoemaker, QB, Thiessen A, Yu PA Pub. Chem. (2019) .Update: Improved Access to Chemical Data. Nucleic Acids Res. 47:D1102-D1109.

[27] Berman H, Battistuz MT, Bhat TN, Bluhm WF, Bourne PE, Burkhardt K, Feng Z, Gilliland GL, lype L, Jain S et al(2002). The Protein Data Bank. Acta Crystallogr. Sect. D: Biol. Crystallogr. 58:899-907.

[28] Waterhouse A, Bertoni M, Bienert S, Studer G, Tauriello G, Gumienny R, Heer FTT, De Beer AP, Rempfer C, Bordoli L, et al. (2018). SWISS-MODEL: Homology Modelling of Protein Structures and Complexes. Nucleic Acids Res;46:W296- W303.

[29] Zhang L, Zhou R (2020). Structural Basis of the Potential Binding Mechanism of Remdesivir to SARSCoV-2 RNA-Dependent RNA Polymerase. J. Phys. Chem. B. 124:6955-6962.

[30] Dallakyan S, Olson AJ (2016).Small-Molecule Library Screening by Docking with PyRx. Methods. Mol. Biol.;1263:243-50.

[31] Wakchaure PD, Ghosh S, Ganguly B (2020) Revealing the Inhibition Mechanism of RNA-Dependent RNA Polymerase (RdRp) of SARS-CoV-2 by Remdesivir and Nucleotide Analogues: A Molecular Dynamics Simulation Study. The Journal of Physical Chemistry B. ;124:10641-10652.

[32] Dassault Systèmes, BIOVIA (2016). Discovery Studio Modeling Environment, Release 2017, San Diego: Dassault Systèmes,.

[33] Wakchaure PD, Velayutham R, Roy KK (2019). Structure investigation, enrichment analysis and structure-based repurposing of FDA-approved drugs as inhibitors of BET-BRD4. Journal of Biomolecular Structure and Dynamics.; 37: 3048-3057.

[34] Si MK, Sen A. \& Ganguly B. (2017) Exploiting hydrogen bonding interactions to probe smaller linear and cyclic diamines binding to G-quadruplexes: a DFT and molecular dynamics study. J. Phys. Chem. Chem. Phys. 19:11474.

[35] Shi D, Heel house DS \& Hurley LH (2001) Quadruplex-Interactive Agents as Telomerase Inhibitors: Synthesis of Porphyrins and Structure-Activity Relationship for the Inhibition of Telomerase. J. Med. Chem. 44:4509-4523.

[36] Bussi G, Donadio D \& Parrinello M (2007) Canonical sampling through velocity rescalingJ. Chem. Phys., 126, 014101. 
[37] Berendsen HJC, Postma JPM, van Gunsteren WF, DiNola A \& Haak JR (1984) Molecular dynamics with coupling to an external bath J. Chem. Phys., 81, 3684-3690.

\section{Figures}

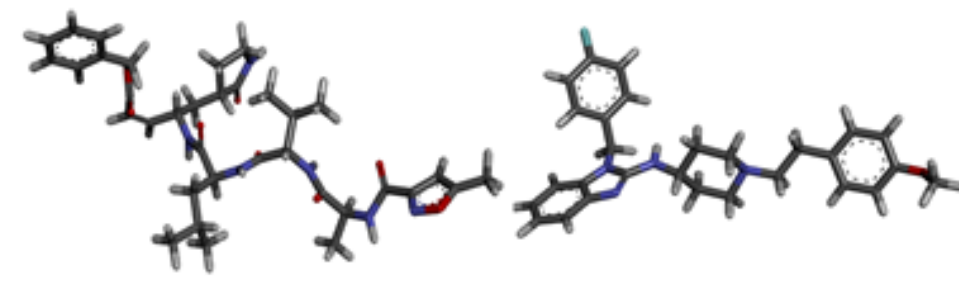

1. N3-4

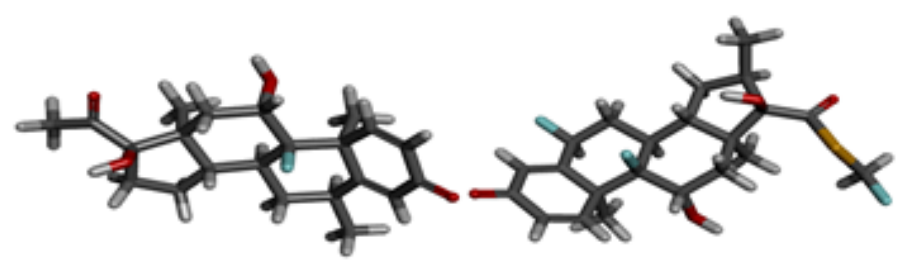

5. Fluorometholone

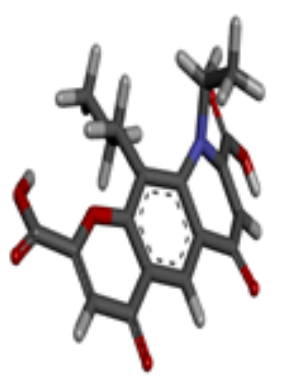

9. Nedocromil

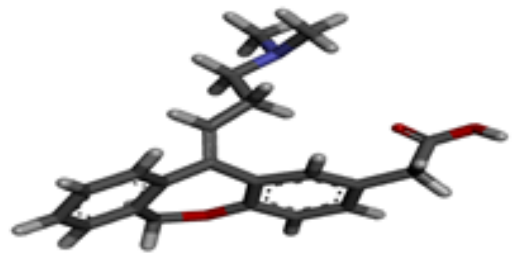

10. Olopatadine

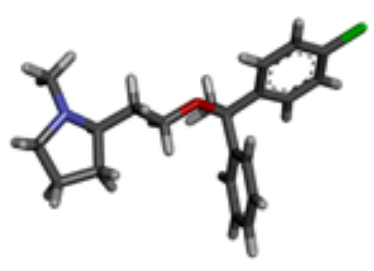

3. Clemastine

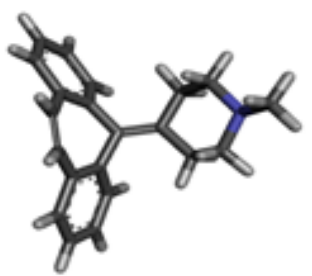

4. Cyproheptadine

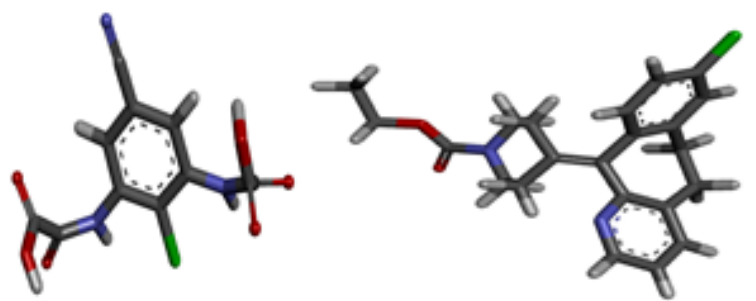

7. lodoxiamide

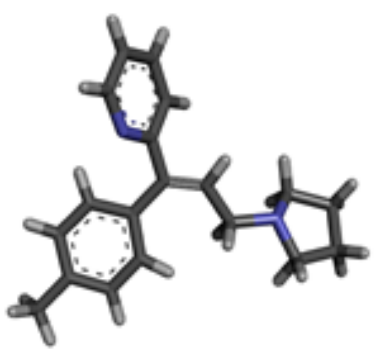

11. Triprolidine

\section{Figure 1}

Structure of anti-allergic drug molecules. 1 is the experimentally reported inhibitor of Covid-19 protein. 
(a)
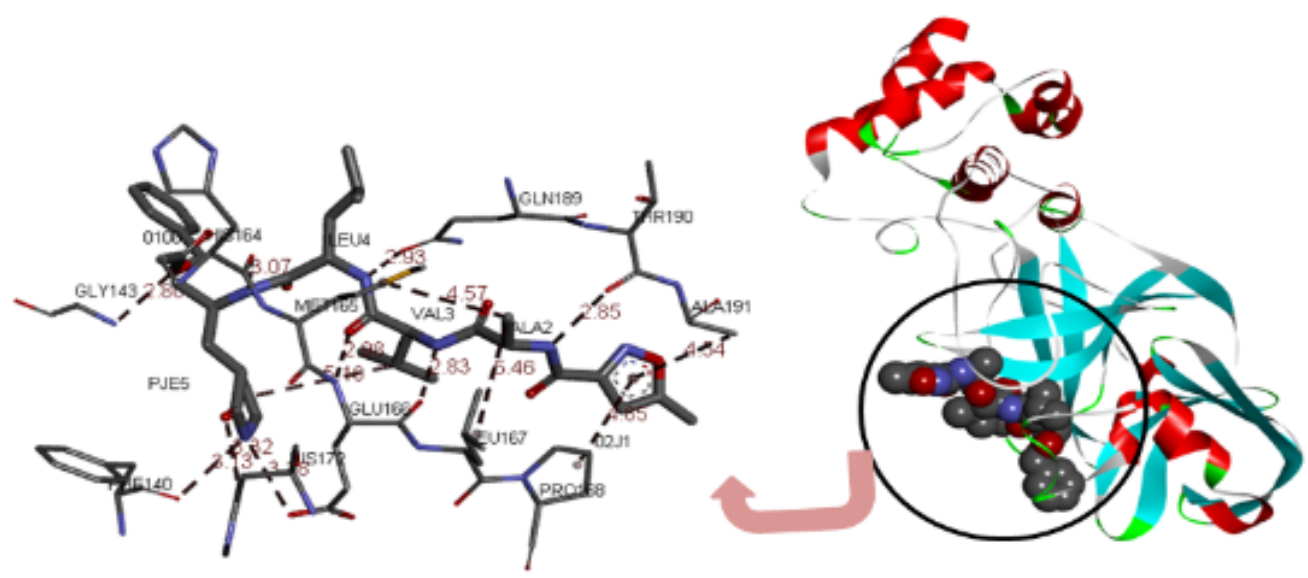

(b)
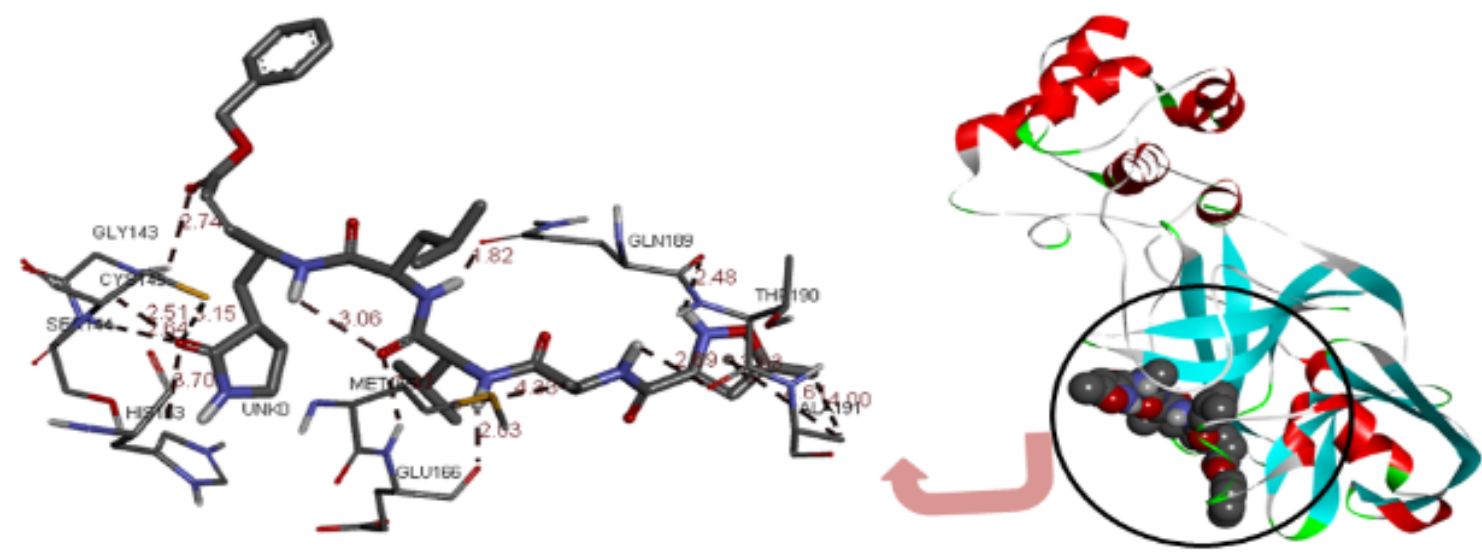

Figure 2

(a) Active site residues interacted with inhibitor N3-4 in crystal structure (b) Active site residues interacted with inhibitor N3-4 in docked structures. 


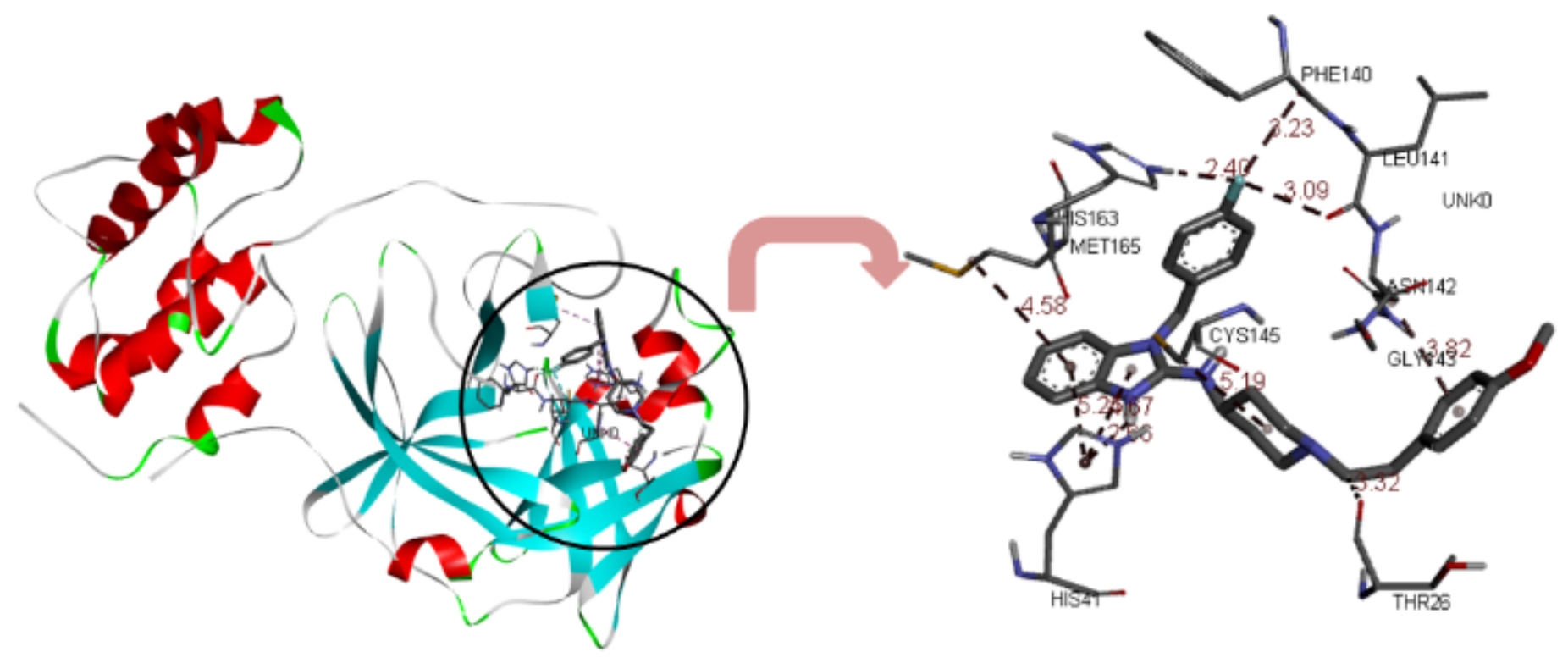

Figure 3

Active site residues of Covid-19 main protease interacted with anti-allergic Astemezole

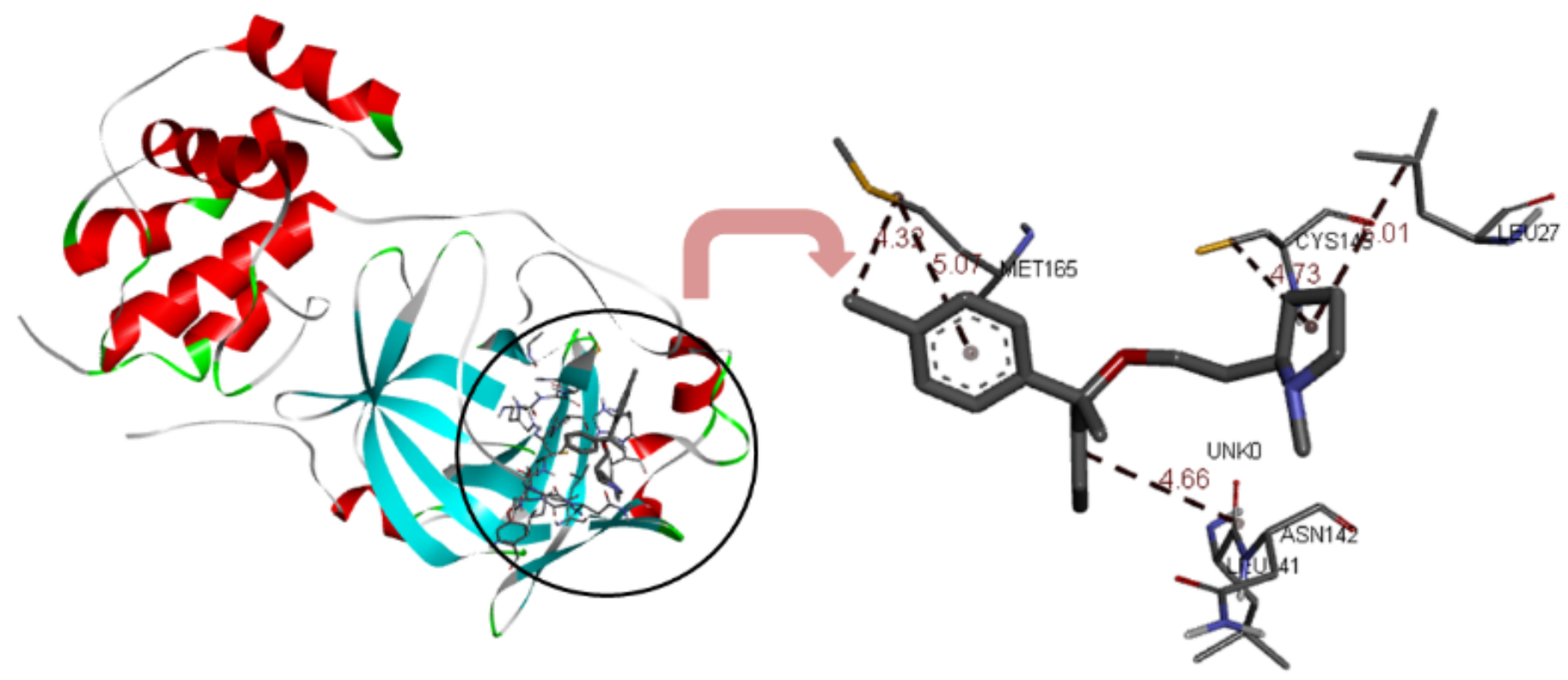

Figure 4

Active site residues of Covid-19 main protease interacted with anti-allergic Clemastine 


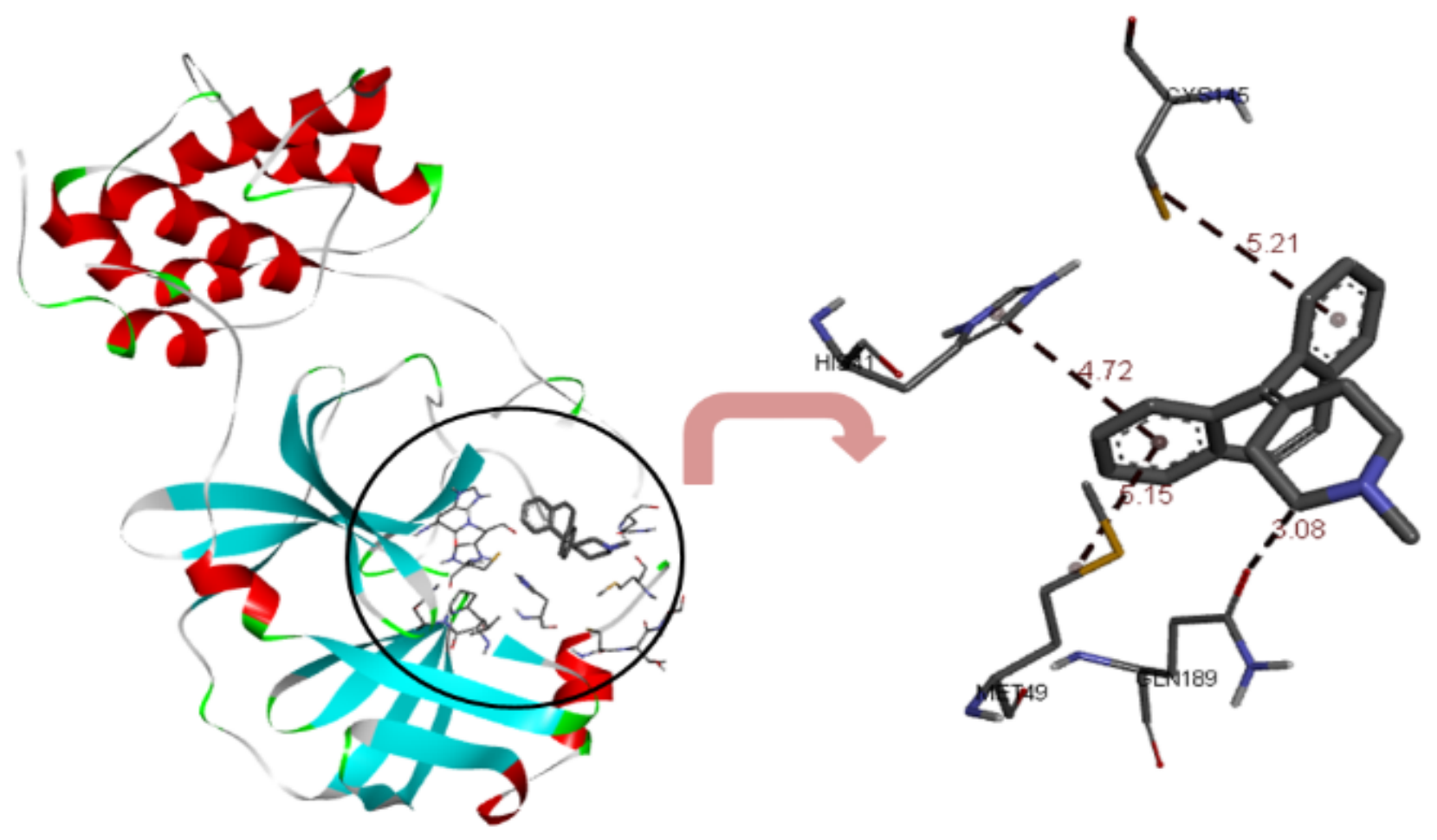

Figure 5

Active site residues of Covid-19 main protease interacted with anti-allergic Crypoheptadiene

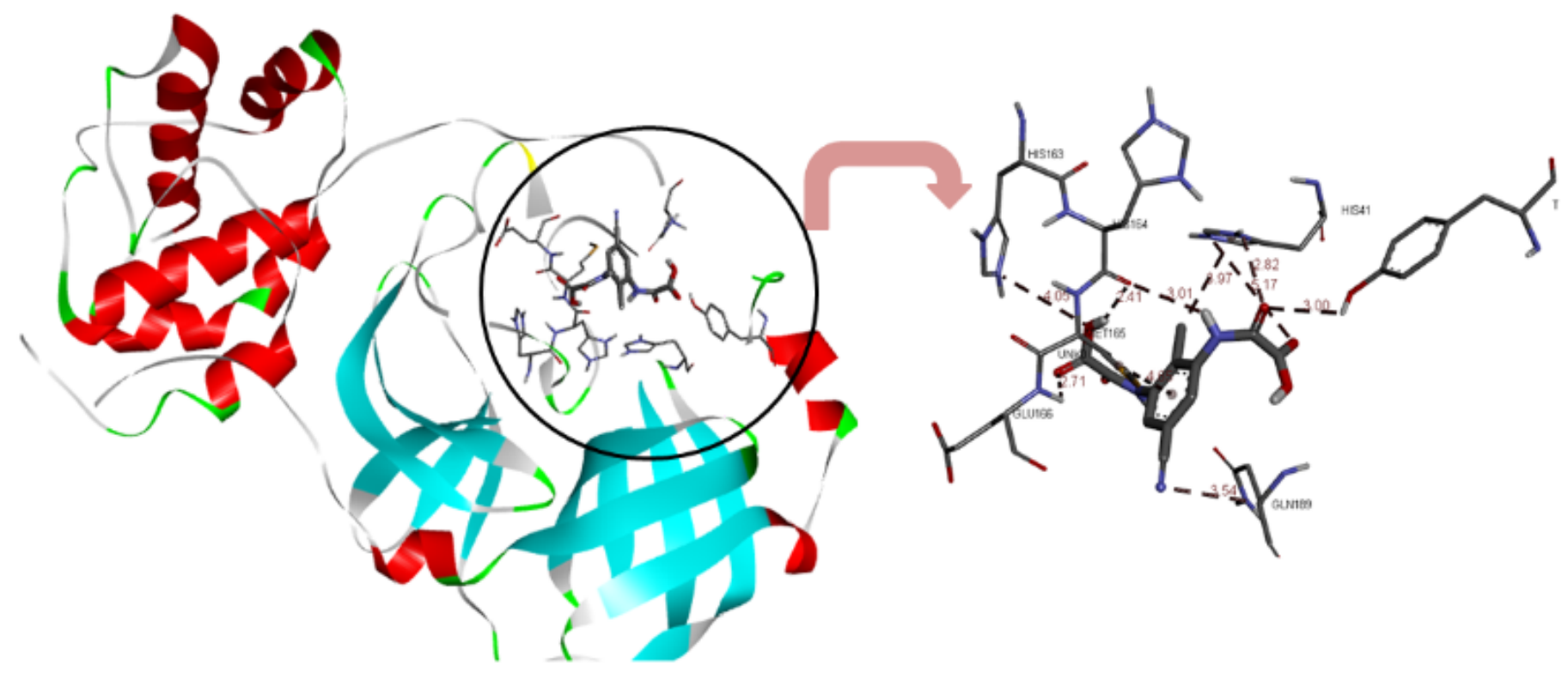

Figure 6

Active site residues of Covid-19 main protease interacted with anti-allergic lodoxamide 


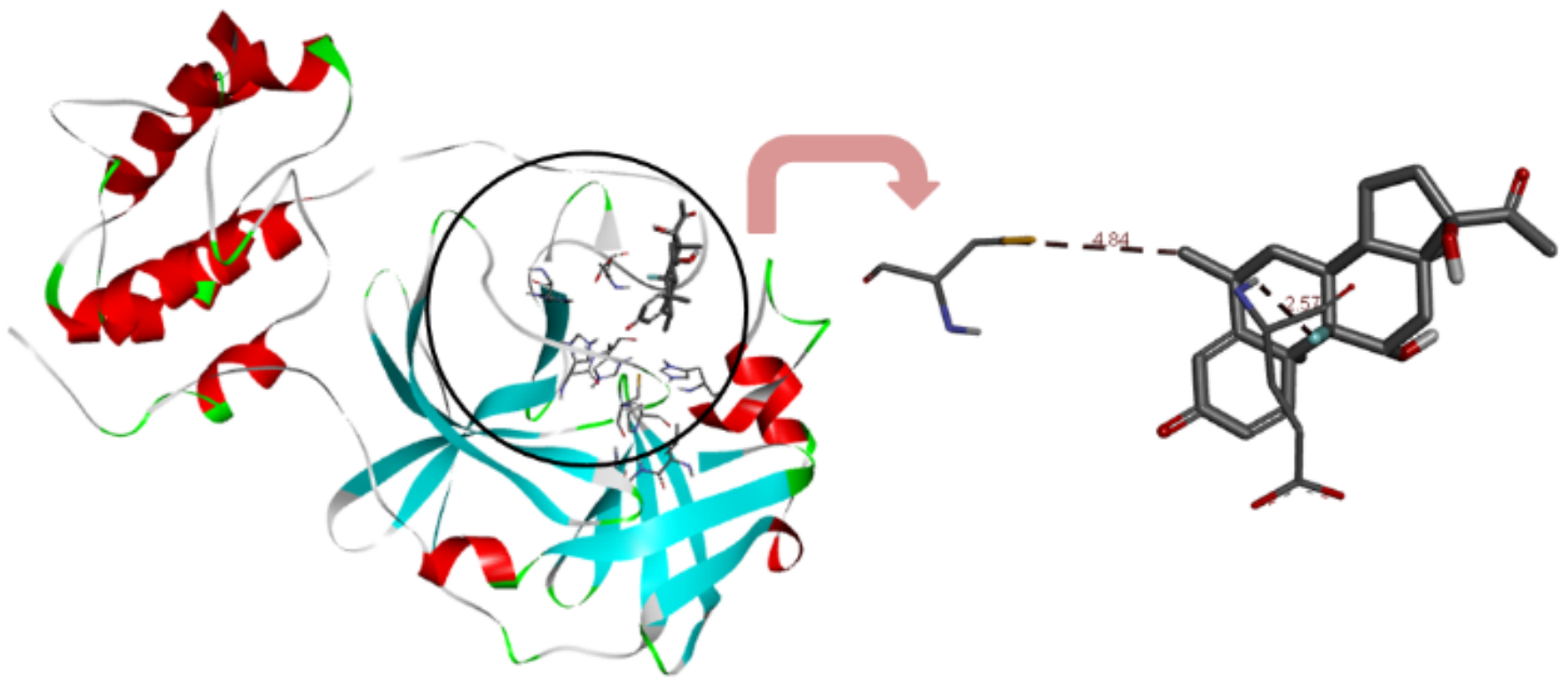

Figure 7

Active site residues of Covid-19 main protease interacted with anti-allergic Fluoromethone

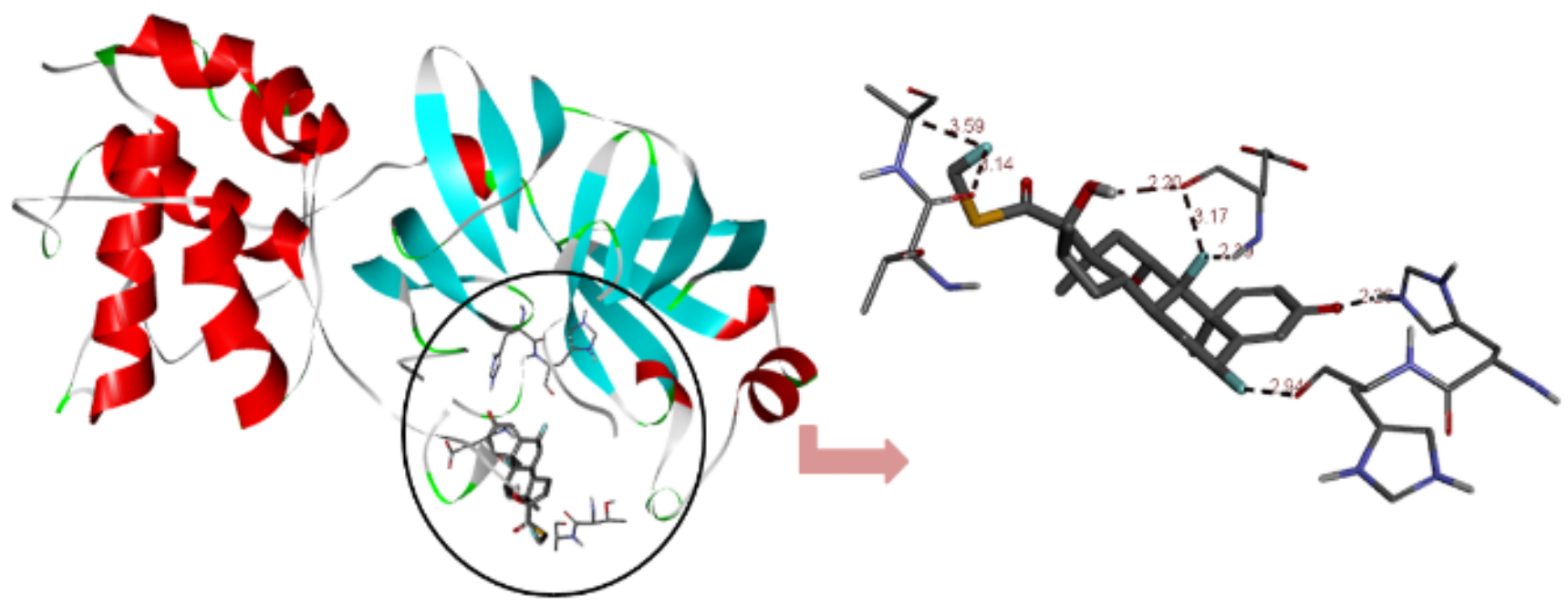

Figure 8

Active site residues of Covid-19 main protease interacted with anti-allergic Fluticasoni 
(a)

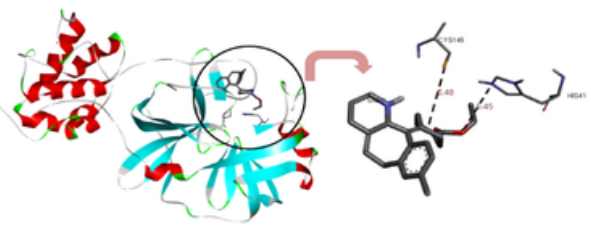

(b)

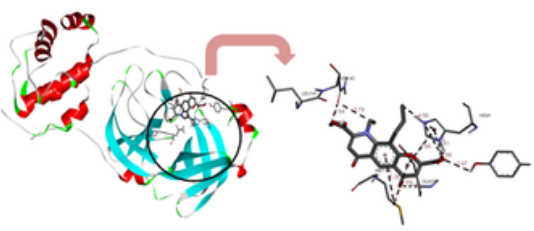

(c)

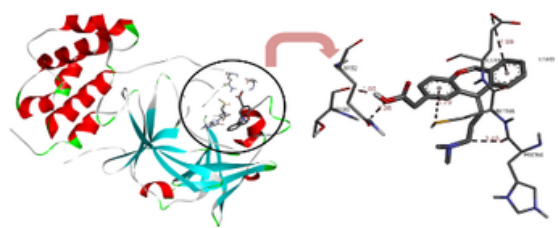

(d)

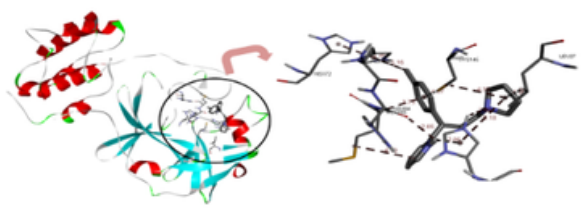

\section{Figure 9}

Active site residues of Covid-19 main protease interacted with anti-allergic (a) loratadiene (b) Nedocromil (c) Olopatadiene (d) Triprolidine

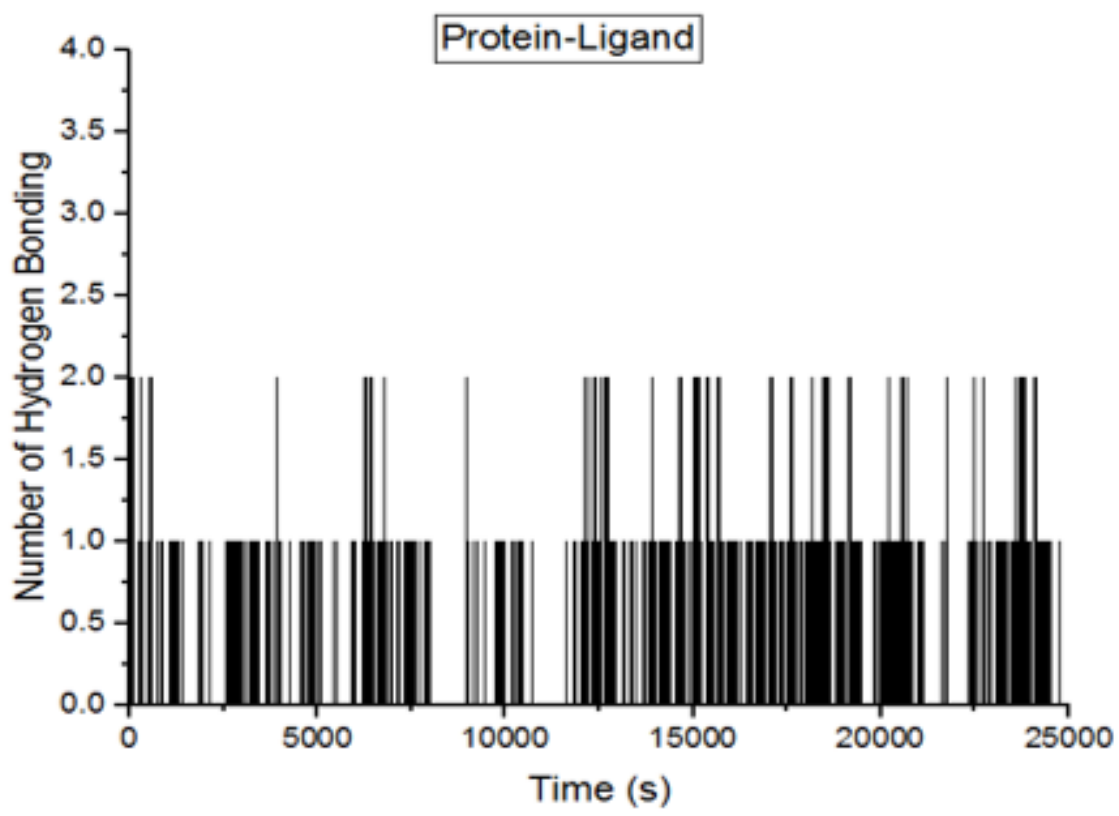

Figure 10 
Number of total hydrogen bonding between Astemizole and Covid-19 protein

(a)

(b)
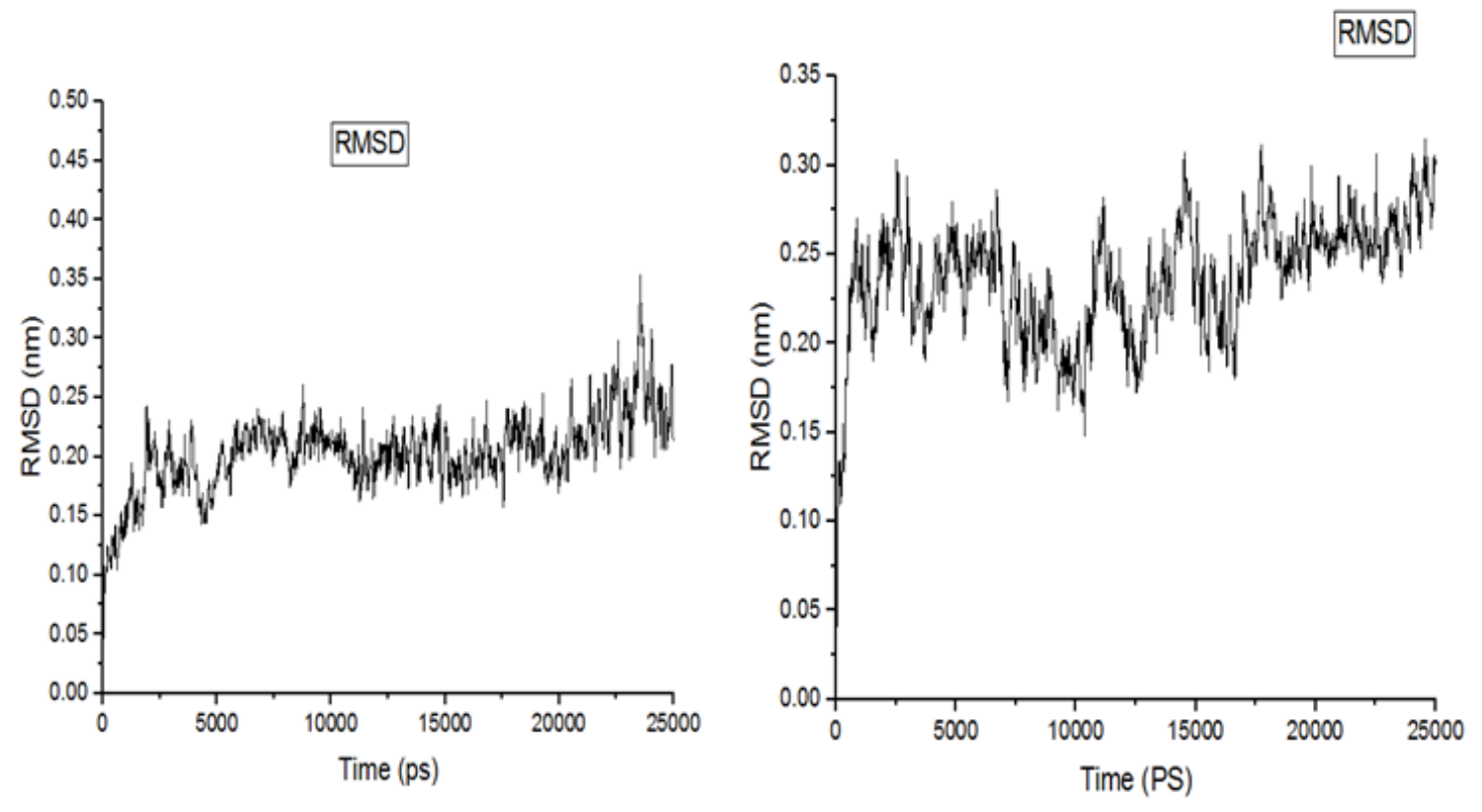

Figure 11

Plot of Root-mean-square deviation (RMSD) of various residues of (a) Covid-19 Protein (b) Covid-19 Protein-Astemizole complex

(a)

(b)
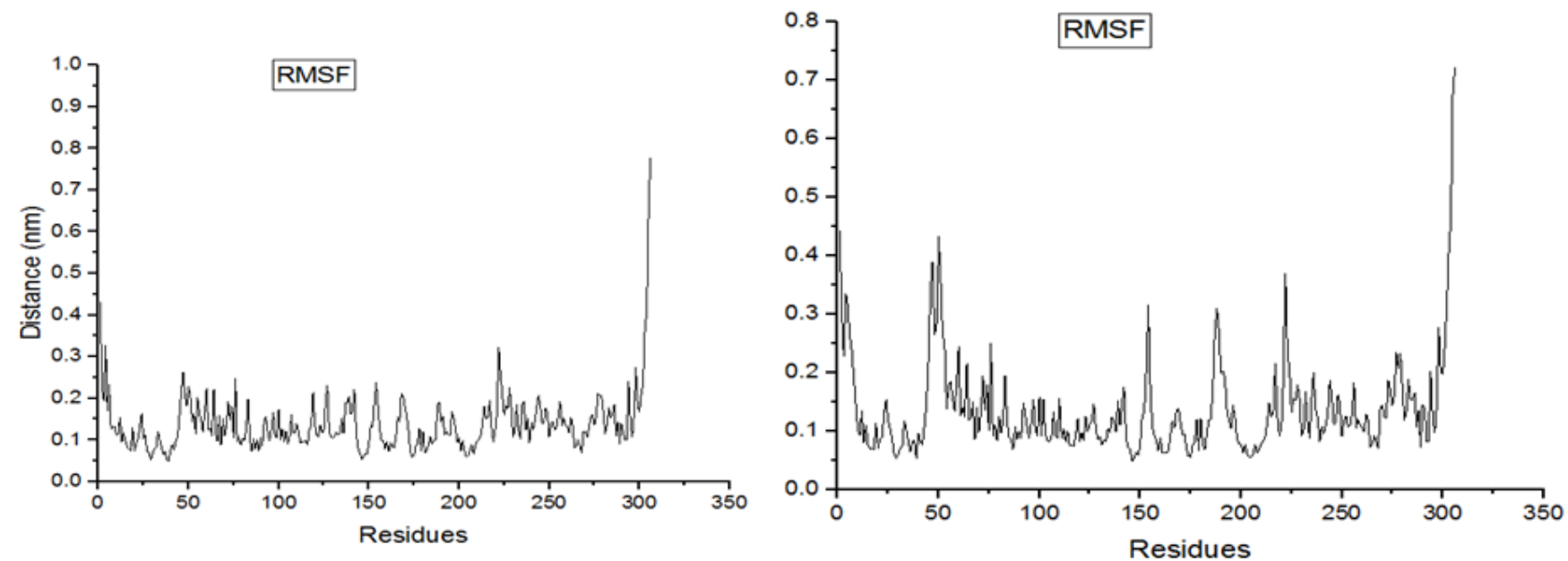
Figure 12

Plot of root mean square fluctuation (RMSF) of various residues of (a) Covid-19 Protein (b) Covid-19 Protein-Astemizole complex with time

(a)

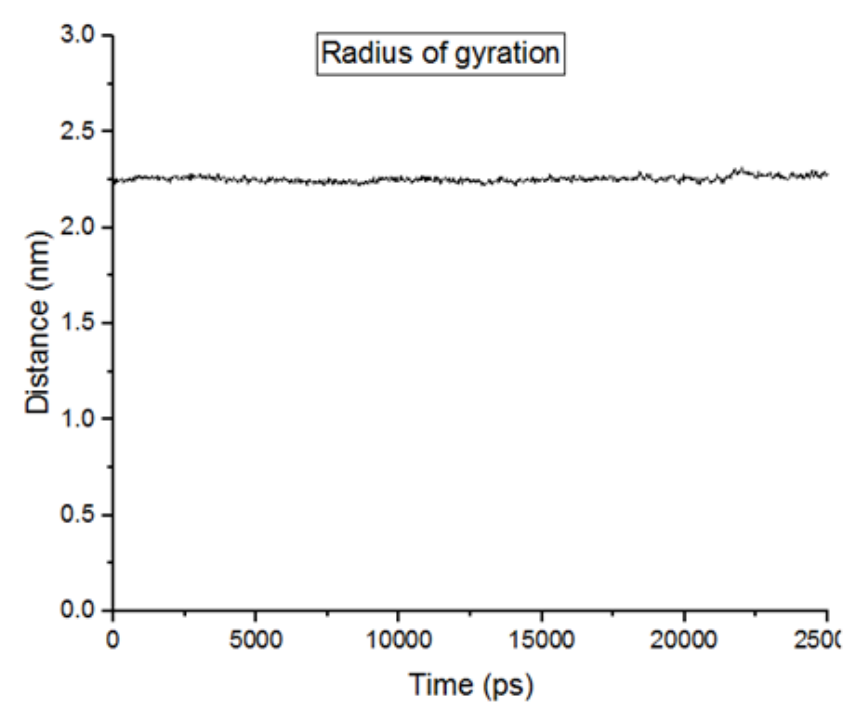

(b)

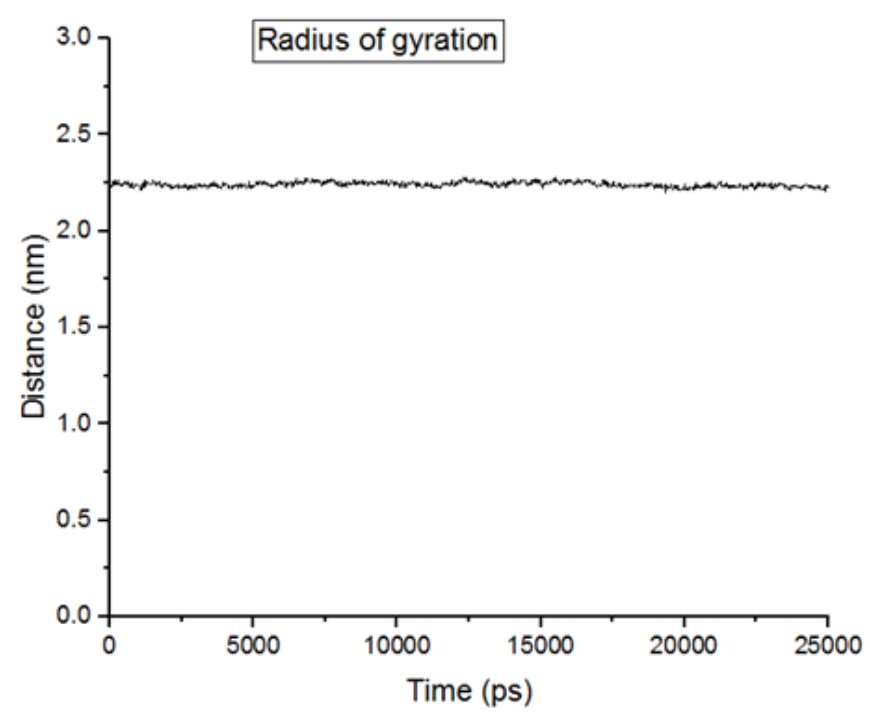

\section{Figure 13}

Plot of radius of gyration of (a) Covid-19 Protein (b) Covid-19 Protein-Astemizole complex with time radius of gyration 
(a)

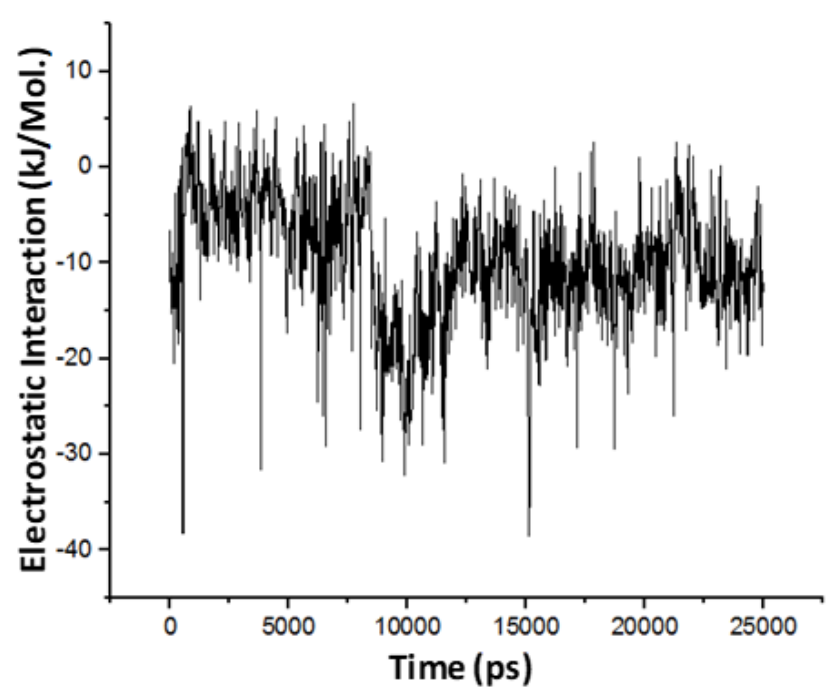

(b)

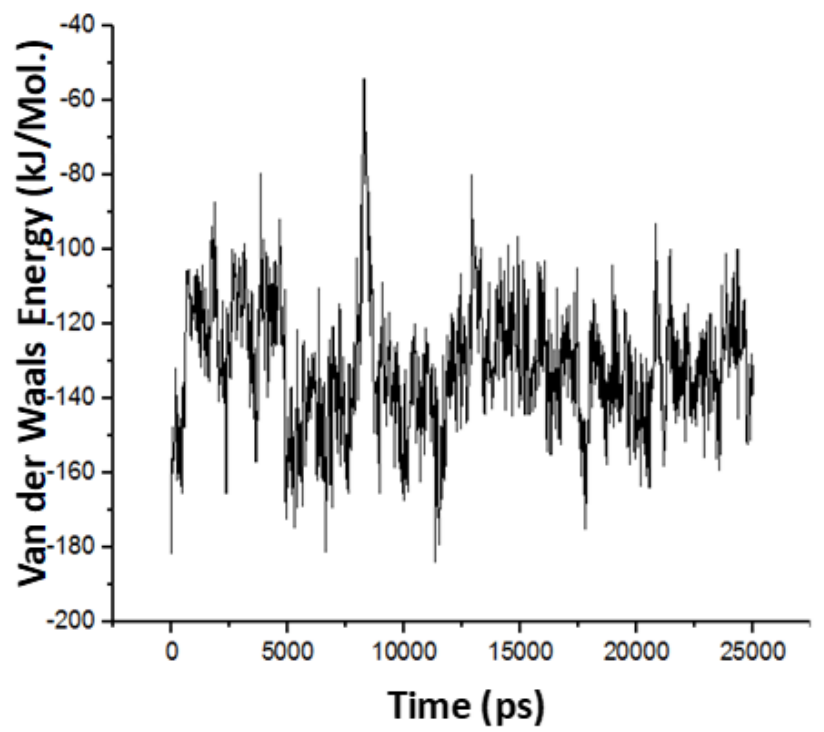

Figure 14

(a) Plot of electrostatic interaction between Astemizole and main protease of Covid-19 with time during MD simulation. (b) Plot of Van der Waals interaction between Astemizole and main protease of Covid-19 with time during MD simulation.

\section{Supplementary Files}

This is a list of supplementary files associated with this preprint. Click to download.

- GraphicalAbstract.docx 